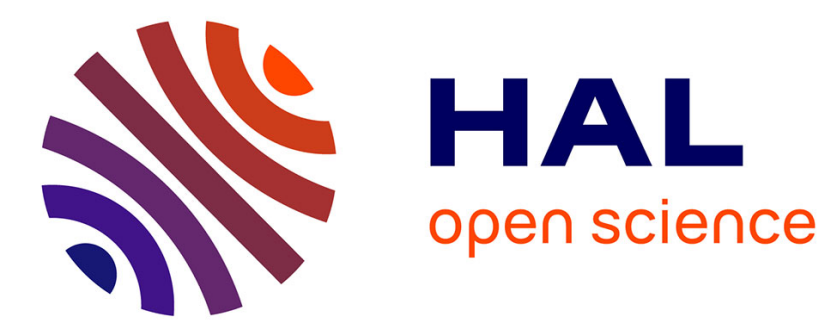

\title{
L'Église et les catholiques de France face à la question migratoire : le grand malentendu ? \\ Vincent Geisser
}

\section{To cite this version:}

Vincent Geisser. L'Église et les catholiques de France face à la question migratoire : le grand malentendu ?. Migrations Société, 2018, 173 (3), pp.3. 10.3917/migra.173.0003 . hal-02110732

\section{HAL Id: hal-02110732 \\ https://hal-amu.archives-ouvertes.fr/hal-02110732}

Submitted on 25 Apr 2019

HAL is a multi-disciplinary open access archive for the deposit and dissemination of scientific research documents, whether they are published or not. The documents may come from teaching and research institutions in France or abroad, or from public or private research centers.
L'archive ouverte pluridisciplinaire HAL, est destinée au dépôt et à la diffusion de documents scientifiques de niveau recherche, publiés ou non, émanant des établissements d'enseignement et de recherche français ou étrangers, des laboratoires publics ou privés.

\section{(이)(\$)}

Distributed under a Creative Commons Attribution - NonCommercial - NoDerivatives| 4.0 


\section{L'ÉGLISE ET LES CATHOLIQUES DE FRANCE FACE À LA QUESTION MIGRATOIRE : LE GRAND MALENTENDU ?}

Vincent Geisser

Centre d'information et d'études sur les migrations internationales | «Migrations Société »

2018/3 $\mathrm{N}^{\circ} 173$ | pages 3 à 13

ISSN 0995-7367

Article disponible en ligne à l'adresse :

https://www.cairn.info/revue-migrations-societe-2018-3-page-3.htm

Distribution électronique Cairn.info pour Centre d'information et d'études sur les migrations internationales.

(C) Centre d'information et d'études sur les migrations internationales. Tous droits réservés pour tous pays.

La reproduction ou représentation de cet article, notamment par photocopie, n'est autorisée que dans les limites des conditions générales d'utilisation du site ou, le cas échéant, des conditions générales de la licence souscrite par votre établissement. Toute autre reproduction ou représentation, en tout ou partie, sous quelque forme et de quelque manière que ce soit, est interdite sauf accord préalable et écrit de l'éditeur, en dehors des cas prévus par la législation en vigueur en France. Il est précisé que son stockage dans une base de données est également interdit. 


\section{L'Église et les catholiques de France face à la question migratoire : le grand malentendu?}

Vincent GEISSER

Depuis leur création, le ClEMI et la revue Migrations Société consacrent assez régulièrement des dossiers thématiques, tables rondes, séminaires et colloques au rapport qu'entretiennent les catholiques à la question migratoire et, sur un plan plus général, à la manière dont les institutions religieuses (chrétiennes, musulmanes, juives, etc.) prennent en charge les phénomènes multiculturels et pluriconfessionnels dans les sociétés démocratiques ${ }^{\mathrm{I}}$. Cette orientation thématique doit beaucoup à la trajectoire historique du Centre et de la revue qui, en dépit du processus de sécularisation des sociétés européennes, ont toujours considéré que les croyants et les pratiquants des différentes confessions présentes dans l'Hexagone et dans l'Union européenne avaient un rôle à jouer dans les différents champs sociaux, notamment celui des migrations internationales, en matière d'actions de solidarité, d'interpellation des pouvoirs publics, mais aussi comme objet d'étude et de réflexion. La profusion des initiatives et des publications du CIEMI sur ces sujets en est la preuve. Et nous continuerons probablement dans les années à venir à nous investir sur ces questions, car les institutions religieuses et les fidèles des différentes confessions ne peuvent être considérés simplement comme des survivants d'une époque révolue, mais constituent aussi des acteurstémoins des conceptions éthiques, philosophiques et des pratiques sociales enracinées dans la modernité, qui influencent de manière déterminante notre relation à l'Autre : l'étranger, le migrant, le réfugié, le minoritaire, etc. De ce point de vue, les animateurs du ClEMI sont convaincus que les catholiques, les protestants, les juifs, les musulmans, les bouddhis-

1. À titre d'exemple, les 18 et 19 janvier 2013, le CIEMI a organisé au Collège des Bernardins un colloque intitulé «Les catholiques et les migrations: histoire, actualité, perspectives 》, dont une partie des communications avait été publiée dans Migrations Société (voir "Les catholiques et les migrations" (dossier), Migrations Société, vol. 24, n 139, janvier-février 2012, pp. 47-266). Le 12 juin 2007, le CIEMI a été à l'initiative d'une table ronde intitulée «Acteurs religieux et laïques dans les quartiers populaires : médiateurs tout terrain et vecteurs de paix sociale? », réunissant un prêtre, un rabbin, un imam, un pasteur protestant et un représentant d'une association laïque. 
tes, les déistes, etc., ont encore des choses à dire sur la question migratoire. Leur parole mérite d'être entendue.

Malgré notre volonté de recueillir, d'analyser et de rendre compte de la diversité des points de vue religieux et éthiques sur la question migratoire, il convient d'admettre que notre démarche compréhensive s'est souvent heurtée à un point aveugle, confortant un «biais institutionnaliste » et une approche surplombante. En effet, dans nos écrits et nos cercles de réflexion, nous nous sommes davantage intéressés aux textes canoniques, aux références théologiques et aux déclarations des autorités religieuses et des porte-parole officiels sur la question de l'immigration, qu'à celui des croyants et des pratiquants ordinaires. Ce biais ne relève pas d'un choix délibéré mais plutôt de notre difficulté à atteindre ces «gens normaux » qui constituent pourtant la base sociale et «bien vivante » des institutions religieuses et des cultes constitués dans la société française d'aujourd'hui. C'est probablement ce constat qui a poussé plusieurs organisations catholiques indépendantes (Service national de la pastorale des migrants et des personnes itinérantes de la Conférence des évêques de France, CCFD-Terre solidaire ${ }^{2}$, Secours Catholique, JRS France ${ }^{3}$ ) à commander à More in Common ${ }^{4}$ et à l'Institut français d'opinion publique (IFOP) une enquête inédite, revêtant un volet quantitatif (sondage par quotas auprès de I 002 individus) et un volet qualitatif (constitution de panels de discussion) sur le rapport des catholiques français à l'immigration. Au-delà des insatisfactions et des frustrations légitimes que peut éprouver le lecteur à l'égard des résultats de ce type d'enquête très formatée, l'étude intitulée Perceptions et attitudes des catholiques de France vis-à-vis des migrants, parue en juin 2018, permet de corroborer des observations établies depuis plusieurs années sur les évolutions affectant la «catholicité française », tout en apportant dans le même temps des éléments nouveaux qui méritent d'être commentés 5 .

Au-delà des résultats détaillés sur le rapport des catholiques à l'immigration et à l'islam ${ }^{6}$, l'enquête révèle d'abord la pluralité de la catholicité hexagonale, c'est-à-dire des «manières d'être catholique » dans la

2. Comité catholique contre la faim et pour le développement.

3. Jesuit Refugee Service.

4. More in Common est une association loi 1901 fondée en 2017 dans l'objectif d'« immuniser nos sociétés contre la tentation du repli identitaire, social et culturel en faisant la promotion de ce que nous avons en commun »: https://www.moreincommon.com/francais/.

5. MORE IN COMMON, Perceptions et attitudes des catholiques de France vis-à-vis des migrants, Paris, juin 2018, 71 p., https://static1.squarespace.com/static/5a70a7c30100277 36a22740f/t/5b180f54f950b74ab7834990/1528303446739/More+in+Common+French+Ca tholics+Report.pdf.

6. Tenant compte du fait que la question migratoire est très fortement liée dans le débat public au «problème musulman », et que de nombreux migrants sont souvent perçus, à tort ou à raison, comme «musulmans », les responsables de l'enquête ont jugé pertinent d'interroger également les catholiques de France sur leur rapport à l'islam et aux musulmans. 
société française de ce début de XXI siècle. Elle remet ainsi en cause l'idée d'un bloc homogène et l'existence d'un habitus catholicus qui structurerait les attitudes, les comportements et les représentations des croyants et des pratiquants ordinaires. Même si une majorité de Français continuent à se définir spontanément comme "catholiques", leur catholicisme ne peut plus être considéré comme une identité à la fois exclusive et totale qui déterminerait leur appréhension des questions sociales et leur vision du monde. En 20I8, les catholiques de France vivent des appartenances multiples et le catholicisme est traversé par de nombreux clivages socioculturels : classistes, générationnels, territoriaux, de genre, et bien sûr politiques et philosophiques, qui contribuent à orienter de manière décisive le rapport à la question migratoire et, au-delà, la relation à l'altérité culturelle et religieuse. Ce constat, déjà établi par des études précédentes 7 , est confirmé par cette nouvelle enquête : «Rappelons d'abord une première évidence : ils [les catholiques de France] ne vivent pas hors du monde. Leur sentiment d'appartenance au catholicisme est l'une des composantes de leur identité, individuelle et collective. Il vient se combiner à leur identité nationale ou locale, à leurs affinités politiques, aux intérêts de leur catégorie socioprofessionnelle, à leur niveau de revenu, à la génération à laquelle ils appartiennent, à leur expérience vécue. La question est de savoir quelle valeur les catholiques accordent à leur foi parmi les composantes de leur identité, et si la foi joue en faveur d'un "nous" inclusif ou au contraire en faveur d'un "nous" exclusif $»^{8}$.

Parce que précisément, les catholiques de France ne forment pas un «isolat social» - y compris les plus pratiquants d'entre eux -, ils n'échappent pas aux discours politico-médiatiques et aux représentations de sens commun sur l'immigration, ayant tendance à amplifier, à l'instar des autres Français, le phénomène migratoire sur un registre dramatique. Les auteurs du rapport parlent ainsi d'un « effet Calais », c'est-à-dire d'une tendance à réduire la complexité des réalités migratoires à ce cas particulier: "On retrouve ainsi chez les catholiques ce décalage entre la réalité des flux et leur perception déjà observé dans la population globale : alors que l'INSEE 9 donne à voir un nombre d'arrivées relativement stable depuis plusieurs années, et un solde migratoire quasiment identique depuis 1975, le traitement médiatique de la question et son omniprésence dans le débat politique créent un sentiment de submersion, parfois décrit comme "l'effet Calais" $»^{\mathrm{I}}$.

7. RICHARD, Jean-Luc, "Les catholiques, l'immigration étrangère et les tentations racistes en France. Quelques apports d'enquêtes d'opinion et de données macro-sociales", Migrations Société, vol. 24, n 139, janvier-février 2012, pp. 253-266.

8. MORE IN COMMON, Perceptions et attitudes des catholiques de France vis-à-vis des migrants, op. cit. (voir p. 6).

9. Institut national de la statistique et des études économiques.

10. MORE IN COMMON, Perceptions et attitudes des catholiques de France vis-à-vis des migrants, op. cit. (voir p. 18). 
Mais, outre qu'elle n'est ni homogène, ni univoque, cette relation des catholiques de France à la question de l'immigration relève moins d'un « choc frontal » que d'une ambivalence permanente. Celle-ci se manifeste par un tiraillement constant entre des tendances anxiogènes (peur de l'invasion immigrée) et des tendances à l'altruisme, ces dernières se traduisant par une forme de bienveillance pour le sort des déplacés, des réfugiés et des migrants en général. On pourrait ainsi parler à propos des catholiques de France d'un conservatisme compassionnel ${ }^{\mathrm{II}}$ : "Mais la controverse masque l'essentiel. On en oublie ce qui affleure parmi les catholiques : une ambivalence entre l'hospitalité due à l'étranger et l'inquiétude face à son étrangeté $»^{\mathrm{I2}}$.

Sur ce dernier point, les résultats de l'étude réalisée par More in Common tendraient à montrer que l'intégration institutionnelle dans les milieux de l'Église, si elle ne supprime pas totalement l'ambivalence des catholiques de France à l'égard des questions «migratoire » et «musulmane », contribuerait à réduire les tensions et surtout les expressions verbales de haine. En somme, même si cela peut surprendre certains lecteurs, les catholiques pratiquants et messalisants expriment moins fréquemment une forme de xénophobie et/ou d'islamophobie larvées que les catholiques détachés de l'Église. Il y aurait donc bien un « effet institution » qui viendrait atténuer les manifestations d'hostilité et de rejet à l'égard des immigrés et des musulmans, ou des deux à la fois, c'est-à-dire des immigrés musulmans. Toutefois, ces «peurs catholiques » ne disparaissent pas complètement, en raison de l'actualité du «terrorisme islamique » et du sort dramatique des chrétiens d'Orient, victimes ces dernières années de la violence des groupes djihadistes. Les catholiques pratiquants sont paradoxalement davantage ouverts sur l'islam, mais - et c'est important de le souligner - ils ont généralement peur des migrants musulmans en raison de leur provenance et de leurs préoccupations concernant le devenir des chrétiens d'Orient et le terrorisme djihadiste. En somme pour eux, les musulmans intégrés ne posent pas de problème particulier, mais les migrants musulmans sont perçus comme une population à risque, ce qui n'empêche pas qu'il faille les accueillir: "Si deux catholiques sur trois estiment que l'influence de l'islam est de plus en plus importante en France, leur perception des musulmans est nuancée. Seul $24 \%$ d'entre eux jugent l'islam incompatible avec la société française. Une majorité relative ne se sent pas radicalement différente des musulmans : $47 \%$ estiment qu'ils ont des valeurs similaires à leurs propres valeurs. Un grand nombre de catholiques refuse également d'en faire un motif de rejet ou d'hostilité à l'accueil : $55 \%$ sont en désaccord avec l'affirmation selon laquelle "c'est un

11. RICHET, Isabelle, "Quand la religion s'engage en politique", Mouvements, vol. $5, n^{\circ} 30$, 2003, pp. 13-20 (voir p. 19).

12. MORE IN COMMON, Perceptions et attitudes des catholiques de France vis-à-vis des migrants, op. cit. (voir p. 62). 
problème que la majorité des migrants venant en France soient musulmans" ${ }^{13}$. Sur ce point aussi, les positionnements des catholiques de France sont à la fois complexes, évolutifs et ambivalents. Dans tous les cas, l'intégration institutionnelle dans l'Église jouerait plutôt en faveur de la tolérance, même s'il convient de parler davantage de «tolérance froide $»^{14}$.

Cet « effet institution » sur les perceptions des catholiques de France à l'égard du fait migratoire et du fait musulman peut se mesurer à deux échelons institutionnels différents qui renvoient en fait à deux niveaux distincts d'intégration à l'univers catholique : "par le haut », c'est-à-dire la fidélité des catholiques ordinaires au message pontifical et des évêques, et «par le bas », à savoir leur degré d'implication dans la vie locale des paroisses. On pourrait rajouter un troisième niveau d'intégration dit « horizontal » qui repose, lui, sur le réseau des associations humanitaires catholiques, très développé dans l'Hexagone et qui représente un autre visage de la catholicité française ${ }^{15}$. Sur ce plan, les résultats de l'étude réalisée par More in Common sont particulièrement éclairants sur la pluralité de la catholicité française et le rapport différentiel à l'Église comme institution socialisatrice et pourvoyeuse de sens.

Concernant le premier niveau d'intégration (la réceptivité des croyants ordinaires au message de la hiérarchie catholique), nous avons fréquemment rendu compte dans les éditoriaux et les articles de Migrations Société des positions plutôt avant-gardistes de l'Église sur la question migratoire et à l'égard des religions monothéistes (juive et musulmane) qui contrastent avec ses positions très conservatrices sur les questions de mœurs et de sexualité. Loin des clichés et des idées reçues d'une Église réactionnaire, repliée sur une identité étriquée et figée, hostile, sinon méfiante à l'égard de toute forme d'altérité culturelle et confessionnelle, l'institution catholique, via les messages des papes qui se sont succédé, très largement relayés à l'échelon des nations par les conférences épiscopales, développe depuis plus de quarante ans une «théologie de l'accueil et de l'altérité » qui n'a rien à envier aux programmes des partis « progressistes ", des associations des droits de l'homme ou des organisations de solidaritér ${ }^{6}$. D'aucuns pourraient accuser l'Église de paternalisme à l'égard des migrants et des minorités religieuses, mais celui-ci a très largement évolué vers un altruisme assumé et revendiqué, notamment depuis

13. Ibidem (voir p. 11).

14. Selon I'expression de CORROLER, Catherine, "Une 'tolérance froide' pour I'islam", Libération, 6 octobre 2003.

15. À titre d'illustration, voir l'article de FRESNOZA-FLOT, Asuncion, "Le rôle de l'Église et des associations dans la structuration de la population immigrée philippine en France", Migrations Société, vol. 21, n 123-124, mai-août 2009, pp. 65-82.

16. COSTES, André, "L'Église catholique dans le débat sur l'immigration", Revue européenne des migrations internationales, vol. $4, \mathrm{n}^{\circ} 1-2,1^{\text {er }}$ semestre 1988, pp. 29-48. 
l'encyclique Pacem in Terris édictée en 1963 par le Pape Jean XXIII ${ }^{17}$ et qui sera reprise plus tard, sous le Pape Paul Vl, dans la Constitution Gaudium et Spes du Concile Vatican II : «Pour atteindre ces fins, les institutions de la communauté internationale doivent, chacune pour sa part, pourvoir aux divers besoins des hommes aussi bien dans le domaine de la vie sociale (alimentation, santé, éducation, travail s'y rapportent), que pour faire face à maintes circonstances particulières qui peuvent surgir ici où là : par exemple, la nécessité d'aider la croissance générale des nations en voie de développement, celle de subvenir aux misères des réfugiés dispersés dans le monde entier, celle encore de fournir assistance aux émigrants et à leurs familles $»^{18}$.

Depuis son élection le I3 mars 2013, le Pape François est allé encore plus loin dans cette mise en œuvre de la "théologie de l'altérité », en faisant bouger les lignes, en secouant les consciences catholiques endormies et en développant une critique radicale de ce qu'il appelle « la mondialisation de l'indifférence ${ }^{\mathrm{I}}{ }^{\mathrm{9}}$, dont les migrants et les réfugiés seraient à la fois les symboles et les victimes ${ }^{20}$. Nul doute que, pour le Pape François, la figure du migrant et du réfugié est par excellence l'incarnation contemporaine de la figure du pauvre: «Nous, le peuple de ce continent, nous n'avons pas peur des étrangers, parce que la plupart d'entre nous étaient autrefois des étrangers. Je vous le dis en tant que fils d'immigrés, sachant que beaucoup d'entre vous sont aussi des descendants d'immigrés ${ }^{2 \mathrm{I}}$.

Mais comment les catholiques de France en général, et les catholiques pratiquants en particulier, reçoivent-ils le discours papal très critique à l'égard de la gestion sécuritaire des flux migratoires, et ses gestes publics en faveur des personnes migrantes (parmi lesquelles l'on trouve beaucoup de réfugiés $)^{22}$ ? Ce $«$ Pape qui dérange $»^{23}$ sur les questions sociales, et en particulier sur la question migratoire, suscite-t-il de l'adhésion ou, au contraire, du rejet dans la base catholique? Sur ce point, l'étude de More in Common apporte des éléments de réponse nuancés : le discours du Pape François, sans forcément susciter une approbation unanime chez les

17. PAPE JEAN XXIII, Pacem in Terris [Paix sur la Terre : sur l'établissement d'une paix universelle dans la vérité, justice, charité et liberté], Encyclique, $1^{\text {er }}$ avril 1963, http://www.vatican.va/holy_father/john_xxiii/encyclicals/documents/hf_j-xxiii_enc_110419 63_pacem_fr.html.

18. PAPE PAUL VI, Gaudium et Spes [Constitution pastorale sur l'Église dans le monde de ce temps], 7 décembre 1965, http://www.vatican.va/archive/hist_councils/ii_vatican_council/ documents/vat-ii_cons_19651207_gaudium-et-spes_fr.html.

19. RIVA, Virginie, Ce pape qui dérange, Ivry-sur-Seine : Éditions de L'Atelier, 2017, 173 p.

20. GEISSER, Vincent, "François, pape des migrants?", Migrations Société, vol. 29, $n^{\circ} 167$, janvier-mars 2017, pp. 3-12.

21. Extrait du discours du Pape François devant le Congrès américain, cité par GEISSER, Vincent, "François, pape des migrants ?", art. cité (voir p. 6).

22. En témoignent ses visites à Lampedusa en juillet 2013, à Lesbos en avril 2016 (il rentre au Vatican avec 12 réfugiés syriens), ou encore le lavement des pieds de différents migrants lors du Jeudi Saint en 2016

23. RIVA, Virginie, Ce Pape qui dérange, Paris : Éditions de l'Atelier, 2017, 173 p.

Vol. $30, n^{\circ} 173$

juillet - septembre 2018 
catholiques ordinaires - notamment chez ceux les mieux intégrés dans les sphères de l'Église - les incite à modérer leurs attitudes d'hostilité à l'égard des migrants et à s'engager davantage dans les actions de solidarité. Selon les auteurs du rapport, il existe donc incontestablement «un effet Pape François », même si celui-ci tend à se limiter aux catholiques pratiquants et conservateurs, les catholiques libéraux et multiculturalistes étant déjà convaincus par la nécessité d'accueillir des migrants et, à l'opposé, les catholiques nationalistes rejetant avec force une orientation papale perçue comme pro-immigrés et islamophile, menaçant à terme l'identité catholique de la France et de l'Europe ${ }^{24}$. En ce sens, ce sont chez les croyants les mieux intégrés dans les réseaux catholiques que l'« effet Pape François » est le plus probant : "Cela laisse sous-entendre que c'est parmi les catholiques conservateurs que joue l'"effet Pape" : à défaut de les convaincre, ou d'être l'unique prescripteur, il contribue à cadrer en partie leur positionnement, et à les arrimer du côté de l'hospitalité. L'engagement de l'Église en faveur de l'accueil contribue donc d'une part à limiter le nombre d'indécis et d'autre part à contenir la proportion de catholiques hostiles à l'accueil $»^{25}$. Pour conclure sur ce registre de l'intégration «par le haut» des catholiques (allégeance au Pape et aux évêques), l'on peut dire qu'elle joue plutôt en faveur d'une forme de retenue, voire d'autocensure: les catholiques pratiquants refusent de cautionner les discours virulents de l'extrême droite sur les questions d'immigration et d'islam ${ }^{26}$. Mais cette attitude de réserve ne saurait être interprétée comme une adhésion claire et franche aux discours du Pape François et des évêques en faveur d'une politique migratoire plus humaniste et d'un dialogue égalitaire avec les musulmans. Si le Pape et les évêques de France sont depuis longtemps dans le registre de la solidarité, les catholiques ordinaires tendent à rester cantonnés au registre de la charité, c'est-à-dire de la compassion chrétienne à l'égard du pauvre et du migrant. En somme, une majorité de catholiques de France affirment respecter le message du Pape sur l'immigration et l'islam davantage par conformisme à l'égard de l'autorité pontificale que par réelle conviction. Signalons également un noyau dur franchement hostile au discours d'ouverture du Pape François, qui est certes largement minoritaire dans l'Église, mais très présent sur les réseaux sociaux et dans certains cercles chrétiens, et que l'on peut définir comme une minorité active catholique anti-immigrés, anti-islam et attachée à l'identité européenne du catholicisme ${ }^{27}$. En France, par exemple, cette minorité active se retrouve parmi le lectorat de l'hebdomadaire

24. TINCQ, Henri, La grande peur des catholiques de France, Paris : Éd. Grasset, 2018, 208 p.

25. MORE IN COMMON, Perceptions et attitudes des catholiques de France vis-à-vis des migrants, op. cit. (voir p. 23).

26. Ce n'est pas le cas des « catholiques nationalistes » qui, eux, ne cachent pas leur posture anti-islam et anti-immigrés.

27. FOURQUET, Jérôme, À la droite de Dieu, Paris : Les Éditions du Cerf, 2018, 176 p. 
Valeurs Actuelles et se reconnaît dans des intellectuels propagandistes comme Laurent Dandrieu ${ }^{28}$.

Le second niveau d'intégration («par le bas ») pourrait être qualifié de « paroissial»: il renvoie à la participation effective des catholiques de France à la vie de leur paroisse et aux institutions chrétiennes de proximité. Comme le rappelle Luca Marin, «le mot "paroisse", aujourd'hui paradoxalement synonyme d'installation stable d'une église, vient du grec "parồkia", signifiant "séjour en terre étrangère", "exode" et témoigne d'un moment historique où les chrétiens se réunissaient dans un lieu écarté du centreville $\gg^{29}$. Or, précisément sur ce point, l'étude réalisée par More in Common apporte un résultat intéressant: en ce début de $\mathrm{XXI}^{\mathrm{e}}$ siècle, l'intégration paroissiale des catholiques de France n'a pas complètement disparu et jouerait même en faveur d'une attention des fidèles pour le sort des migrants et des réfugiés. Ainsi, les catholiques les plus impliqués dans la vie paroissiale sont aussi ceux qui se sentent parmi les plus concernés par la question de l'immigration: "[l'étude] souligne aussi combien les paroisses demeurent des points de repère essentiels, quand bien même elles sont moins fréquentées qu'auparavant. Les catholiques doutent du monde et d'euxmêmes, de leur identité et de leur capacité à nouer un contact durable avec l'altérité, mais ils ne doutent pas du bien-fondé de leur foi, qui reste un moteur de leur manière d'être au monde $\aleph^{30}$. Malgré l'urbanisation croissante ${ }^{3 \mathrm{I}}$, les recompositions territoriales, la dépersonnalisation des relations entre pratiquants et prêtres et le moindre ancrage local de l'Église, « la paroisse demeure un carrefour d'engagement pour les catholiques ${ }^{32}$, notamment en direction des migrants et des réfugiés.

Il conviendrait probablement de rajouter un troisième niveau d'intégration que l'on pourrait définir comme « horizontal», renvoyant au réseau des associations et des organisations catholiques qui, dans le sillage de l'Église, œuvre en faveur des désœuvrés, des migrants et des réfugiés. C'est d'ailleurs une partie des partenaires de ce réseau qui est commanditaire de l'enquête réalisée par More in Common. Là aussi, contrairement à l'image caricaturale d'un repli à la fois individualiste, anxiogène et égoïste des catholiques de France, l'enquête tend à montrer que les croyants pratiquants continuent à s'engager dans les associations

28. DANDRIEU, Laurent, Église et immigration: le grand malaise. Le pape et le suicide de la civilisation européenne, Paris : Presses de la Renaissance, 2017, 288 p.

29. MARIN, Luca, "Approches théoriques et réalités de terrain", Migrations Société, vol. 24, $n^{\circ} 139$, janvier-février 2012, pp. 47-56 (voir p. 50).

30. MORE IN COMMON, Perceptions et attitudes des catholiques de France vis-à-vis des migrants, op. cit. (voir p. 7).

31. CHATELAN, Olivier, "Les catholiques et I'urbanisation de la société française", Vingtième Siècle. Revue d'histoire, vol. 111, $\mathrm{n}^{\circ} 3,2011, \mathrm{pp} .147-158$.

32. MORE IN COMMON, Perceptions et attitudes des catholiques de France vis-à-vis des migrants, op. cit. (voir p. 7). 
humanitaires et solidaires, ne serait-ce que de manière ponctuelle sous la forme de dons ou de participations à des actions de charité en faveur des migrants : "En revanche, leur sentiment d'appartenance à la communauté ecclésiale a un effet manifeste sur leur engagement. Les pratiquants donnent plus : $22 \%$ ont fait un don financier à une association qui vient en aide aux migrants, contre II \% des non-pratiquants. Ils font plus de bénévolat en faveur des personnes déplacées : c'est le cas de $13 \%$ d'entre eux, contre $6 \%$ des nonpratiquants. Et le fait qu'un quart des pratiquants interrogés déclarent avoir accueilli un ou plusieurs migrants dans leur paroisse montre que l'appel de l'Église a bien été relayé et qu'il s'est traduit en solidarités concrètes. La foi est bien un levier pour l'engagement : $40 \%$ des pratiquants l'invoquent en premier pour justifier leur action en faveur des migrants ${ }^{33}$. Au regard de ces résultats, l'on peut conclure que le champ humanitaire et philanthropique évoluant dans le sillage de l'Église est loin d'être totalement sinistré ; il continue à produire de l'influence sur le plan de la conscientisation et de sensibilisation des catholiques ordinaires aux questions sociales et aux problèmes du monde.

Au terme de leurs analyses, les auteurs du rapport opèrent une distinction entre cinq catégories de catholiques ${ }^{34}$, la question de l'immigration et le rapport à l'altérité religieuse (principalement « musulmane ») jouant ici le rôle de révélateur de la diversité et de la pluralité de la catholicité française qui est loin de constituer, comme nous venons de le voir, un bloc homogène. Il n'est pas le lieu de commenter la pertinence de ces catégories - nous renvoyons le lecteur au contenu du rapport —, mais nous les énoncerons très brièvement. En dépit des critiques qu'on peut leur adresser, ces catégories donnent un bon aperçu des différentes manières d'être catholique dans la France de ce début de $\mathrm{XXI}^{\mathrm{e}}$ siècle, remettant en cause nombre d'idées reçues sur le catholicisme hexagonal, trop souvent décrit sur les registres du passéisme, de l'ordre moral ou du « ringardisme». S’il est vrai que certains catholiques de France, prati-

33. Ibidem (voir p. 15).

34. Les cinq catégories dressées par les auteurs de l'étude sont : 1) les catholiques multiculturalistes (jeunes, diplômés, revenus faibles et moyens, électeurs de gauche) favorables à la défense des droits des réfugiés et des immigrés ; 2) les catholiques libéraux (jeunes, très diplômés, revenus élevés, électeurs d'Emmanuel Macron), favorables à la mondialisation et à la liberté de circulation; 3) les nationalistes sécularisés (âge moyen, revenus moyens, non pratiquants, proportion plus élevée d'électeurs Front national/Rassemblement national), hostiles à l'immigration et à l'islam, sentiment d'être lâchés par l'État qui privilégierait les migrants au détriment des Français 4) les catholiques en insécurité culturelle (population plutôt féminine, âgée, davantage pratiquante, électeurs de la droite classique), sentiment de disparition de l'identité française, crispations identitaires mais nuancées par une fidélité à l'autorité papale et au discours du Pape François sur le devoir d'accueil ; 5) les catholiques nationalistes (population âgée, retraitée, plus d'électeurs Front national/Rassemblement national), sentiment d'invasion, attachés aux « racines chrétiennes de la France 》, distinction entre islam = « bonne religion 》 et musulmans = « mauvais comportements », tendances anxiogènes à l'égard du fait migratoire et du fait musulman perçus comme des menaces. Source : Ibidem (voir pp. 12-13). 
quants ou non, sont «travaillés » par un processus de radicalisation idéologique sur les questions de société, en particulier sur celles touchant à l'islam et à l'immigration, et qu'ils se montrent de plus en plus sensibles aux sirènes de l'extrême droite (catégories « nationalistes sécularisés » et «catholiques nationalistes » de l'étude de More in Common), le noyau dur de l'Église catholique aurait plutôt tendance à douter : certes angoissé par les flux de migrants et de réfugiés qu'il a d'ailleurs tendance à surestimer (sensible au thème de la «crise migratoire » et au «syndrome de Calais »), mais restant attentionné aux appels du Pape et des évêques en matière d'accueil, de solidarité et de dialogue avec l'Autre minoritaire (catégorie des «catholiques en insécurité culturelle»). De plus, il continue à exister dans la catholicité française une frange significative de chrétiens engagés auprès des immigrés et des réfugiés, très favorables à l'idée d'une société à la fois multiculturelle et pluriconfessionnelle (catégorie des «catholiques multiculturalistes ») : c'est probablement dans cette frange - même si le rapport ne le dit pas explicitement — que se recrutent les héritiers et les continuateurs des combats historiques des « chrétiens de gauche » ou des « chrétiens progressistes », qui sont certes minoritaires dans l'Église, mais qui restent très actifs dans le réseau associatif catholique et très présents dans les débats publics. Enfin, le catholicisme français est aussi «travaillé » par un phénomène générationnel, celui d'un catholicisme de jeunes diplômés, appartenant généralement aux classes supérieures et aux milieux urbains favorisés (catégorie des «catholiques libéraux») qui développent une vision libérale du monde à la fois au niveau économique (marché), social (égalité/parité) et culturel (diversité), ne percevant pas la question migratoire comme un «danger» ou une «menace», mais davantage comme un facteur d'enrichissement des sociétés modernes. Sans être forcément pratiquants ou militants dans les organisations catholiques de solidarité, on peut penser que ce "catholicisme libéral new age » est aujourd'hui influent dans les milieux intellectuels et les cercles dirigeants et qu'il contribue aussi à réduire le rapport frontal à la question migratoire.

En tant qu'institution pourvoyeuse de sens et levier de mobilisations sociales, l'Église catholique conserve donc un rôle de "guide des consciences », mais moins en matière de reproduction de l'ordre moral ${ }^{35}$ qu'en matière d'ouverture au monde et à l'altérité culturelle et religieuse. Sans vouloir faire de mauvais jeux de mots, l'on serait tenté de dire que l'Église remplit aujourd'hui une fonction de soupape de sécurité à la radicalisa-

35. Bien sûr, ce n'est pas le cas sur les questions de mœurs et de sexualité, à propos desquelles l'Église continue à se présenter comme gardienne de l'ordre moral. Voir à titre d'illustration: O'LEARY, Joseph S, "La théologie catholique face au mariage homosexuel", Cités, vol. 44, n 4, 2010, pp. 27-43 ; LEMOINE, Laurent, "Homosexualité et morale chrétienne aujourd'hui. 'Qui suis-je pour juger ?'", Études, n 10, octobre 2014, pp. 63-73; PORTIER, Philippe, "L'épiscopat dans l'espace politique français", Projet, vol. $342, \mathrm{n}^{\circ} 5$, 2014, pp. 32-39. 
tion idéologique et politique d'une partie des catholiques de France, de plus en plus tentés par les discours xénophobes, anti-migrants et antiislam de l'extrême droite. Sur ce plan, les auteurs du rapport préconisent un certain nombre de recommandations pertinentes, par exemple, la meilleure diffusion du message pontifical du «devoir de solidarité » auprès des catholiques ordinaires; le renforcement de l'intégration paroissiale qui permet d'opérer un rapprochement entre croyants et migrants ; le ciblage des différents publics catholiques afin de mieux répondre à leurs attentes et surtout à leurs angoisses. Mais ne conviendrait-il pas aussi d'expliquer aux catholiques de France que la survie de leur catholicité dépend très largement des migrants, pas en tant que sujets de compassion, mais d'abord en tant que fidèles, croyants et pratiquants, ainsi qu'animateurs et pasteurs de l'Église du XXI ${ }^{e}$ siècle ? Les institutions catholiques ne peuvent plus se passer actuellement de cette présence immigrée en leur sein. Sans les migrants, l'Église est condamnée à mourir. Ce message fort serait sans doute plus convaincant.

Marseille, le 23 septembre 2018 
